\title{
Análisis socioeconómico del mototaxismo en Ocaña, Norte de Santander, Colombia
}

\author{
Socioeconomic analysis of mototaxism in \\ Ocaña, Norte de Santander, Colombia
}

DOI: http://dx.doi.org/10.17981/cultedusoc.11.2.2020.10

Recibido: 6 de abril de 2020 Aceptado:26 de junio de 2020 Publicado: 07 de julio de 2020

\author{
Fernando Barragán Sanchez \\ Universidad Francisco de Paula Santander. Ocaña (Colombia) \\ fbarragans@ufpso.edu.co \\ Jose Manuel Alba Maldonado \\ Universidad Francisco de Paula Santander. Ocaña (Colombia) \\ jmalbam@ufpso.edu.co \\ Merly Zoraida Gelvez Santiago \\ Universidad Francisco de Paula Santander. Ocaña (Colombia) \\ mzgelvezs@ufpso.edu.co
}

Para citar este artículo:

Barragán, F., Alba, J. y Gelvez, M. (2020). Análisis socioeconómico del Mototaxismo en Ocaña, Norte de Santander, Colombia. Cultura, Educación y Sociedad, 11(2). 161-176. DOI: http://dx.doi.org/10.17981/cultedusoc.11.2.2020.10

\section{Resumen}

El moto-taxismo se ha convertido en los últimos años en una fuente de empleo para muchos colombianos; esta actividad se considera ilegal por la legislación por cuanto no reúne los requisitos en torno al servicio público de acuerdo al decreto 2961 de 2006 del Ministerio de Transporte. Por tanto, este artículo se orienta a analizar las causas y consecuencias asociadas al desarrollo de esta actividad en Ocaña Norte de Santander, Colombia; situación que se asume en el marco de la dinámica socioeconómica de una zona de alta vulnerabilidad y muchas carencias. El componente metodológico se aborda desde un enfoque empirista, paradigma cuantitativo, diseño no experimental de tipo descriptivo, para la recolección de la información a través de técnicas como la observación, con un registro de observación empírica. Entre los principales resultados se evidencia que esta actividad es la principal fuente se subsistencia, por cuanto un $51 \%$ de los moto-taxistas son casados y un 90\%, 3 personas en promedio dependen económicamente del moto-taxista. El total de personas que dependen de esta labor equivalen al 9\% de la población total del municipio lo cual es un valor significativo. Destaca como conclusión que muchas de las personas que se dedican a esta labor, lo hacen por la falta de oportunidades laborales en la región, y encuentran en este oficio, el sustento económico para su familia. En el estudio realizado, se evidenció que gran parte de la población Ocañera dedicada al moto-taxismo, conforman familias numerosas, siendo estas la mayoría entre 1 y 5 miembros, los cuales dependen en su mayoría de esta labor.

Palabras clave: Moto-taxismo; economía; estudio; información; ilegal

\begin{abstract}
Moto-taxism has become a source of employment for many Colombians in recent years; this activity is considered illegal by the legislation because it does not meet the requirements for public service according to decree 2961 of 2006 of the Ministry of Transport. Therefore, this article aims to analyse the causes and consequences associated with the development of this activity in Ocaña Norte de Santander, Colombia; a situation that is assumed within the framework of the socio-economic dynamics of an area of high vulnerability and many shortcomings. The methodological component is approached from an empirical approach, a quantitative paradigm, a non-experimental design of a descriptive type, for the collection of information through techniques such as observation, with a register of empirical observation. Among the main results, it is evident that this activity is the main source of subsistence, since $51 \%$ of the motorbike drivers are married and $90 \%, 3$ people on average, depend economically on the motorbike driver. The total number of people who depend on this work is equivalent to $9 \%$ of the total population of the municipality, which is a significant value. The conclusion is that many of the people who work in the industry do so because of the lack of job opportunities in the region, and find in this trade, the economic support for their families. In the study carried out, it was shown that a large part of the Ocañera population dedicated to motor-taxism make up large families, the majority of which are between 1 and 5 members, who depend mostly on this work.

Keywords: Motorcycle taxi; economy; study; information; illegal
\end{abstract}




\section{INTRODUCCIÓN}

El moto-taxismo se ha convertido en los últimos años en una fuente de empleo para muchos colombianos; esta actividad se considera ilegal por la legislación por cuanto no reúne los requisitos en torno al servicio público de acuerdo con el decreto 2961 de 2006 del Ministerio de Transporte, el cual regula la prestación de servicio público en motocicleta. La ciudad de Ocaña, situada en el departamento de Norte de Santander, Colombia, no ha sido ajena a este fenómeno, habitantes de la región ven como el moto-taxismo se ha incrementado exponencialmente en los últimos años.

Según la Alcaldía de Ocaña (2016), el Municipio de Ocaña se encuentra ubicado en la zona Centro Occidental del departamento, y pertenece a la subregión noroccidental, limita por el Oriente con los Municipios de San Calixto, La Playa y Abrego; por el Norte con los municipios de Teorama, Convención y El Carmen, por el Sur con el Municipio de Ábrego y por el Occidente con los Municipios de San Martín y Río de Oro. En el contexto nacional Ocaña hace parte del Norte de Santander ubicado sobre la cordillera Oriental en una zona completamente montañosa, tiene una extensión aproximada de $627.72 \mathrm{Km} 2$ que representa el $2.76 \%$ del área total del Departamento, la cabecera municipal se encuentra a una distancia de $203 \mathrm{Km}$ de la capital del Departamento por la vía Ocaña-Cúcuta, además se comunica con el Departamento del Cesar en la vía Rio de Oro-Aguachica, empalmando con la carretera que va a la Costa Atlántica y hacia el centro del país.

El objetivo del artículo se centró en analizar las causas y consecuencias que llevan a gran cantidad de habitantes de Ocaña a ejercer esta actividad; partiendo de un censo poblacional de las personas que la ejercen, promedio de las ganancias diarias, edades, estrato social, nivel educativo, núcleo familiar, entre otros, y analizando la relevancia que tiene el mototaxismo en la economía de la ciudad.

Una de las mayores dificultades que se presentan en la investigación fue la realización del censo poblacional en la medida que esta actividad al ser ilegal tiene un carácter clandestino debido a el decreto 2961 del 2006 el cual prohíbe el uso de las motocicletas como transporte público, sin embargo, se contó con la experiencia de un grupo de investigadores de la Universidad Francisco de Paula Santander Ocaña del curso de Sociología Ambiental que fueron fundamentales para realizar esta investigación, pues fueron los encargados de buscar la información, realizar entrevistas y el acercamiento con los moto-taxistas.

Para determinar la cantidad de motos utilizadas para el moto-taxismo, se realizó un conteo o numeración de estas, este se hizo en las principales calles de Ocaña donde se ubican moto-taxistas para esperar y recoger pasajeros, para identificarlos, se diseñó una matriz donde predominan algunas características propias de las personas que ejercen esta labor, como el tipo de vestuario (que generalmente es cubriendo casi la totalidad del cuerpo con prendas para evitar quemaduras por el sol), su predisposición y ofrecimiento para llevar pasajeros, portar un casco adicional para el pasajero, entre otros.

Dentro de los hallazgos más importantes en la investigación fue el gran número de personas ejerciendo esta actividad los cuales son cercanos a los cuatro mil, convirtiendo el moto-taxismo en una de las principales fuentes de empleo para la ciudad. Como la asegura Caro y Cotinchara (2018): "El mototaxismo se ha convertido con el paso del tiempo en el principal medio de transporte público para la población e incluso ha llegado a construir 
la más importante actividad económica informal y así dicho servicio se convierte en una alternativa de empleo" (p. 10), donde gran número de personas se benefician de esta labor, destacando el hecho de, que se ha convertido en una actividad ligada a los estratos uno y dos como fuente de sobrevivencia.

Por moto-taxismo, se entiende la labor que ejerce cierto grupo de personas, la cual consiste en transportar en motocicleta a demandantes de movilidad de un lugar a otro, generalmente a zonas urbanas por un valor pecuniario. Rodríguez y González (2017) definen el moto-taxismo como toda "actividad económica que hace parte de la denominada "economía del rebusque" que consiste en el transporte de personas en motos" (p. 26).

El comienzo de moto-taxismo en Colombia surge en el departamento de Córdoba como una práctica de transporte público hace más de 10 años, debido a la falta de oportunidades laborales para profesionales de todo orden, desempleados que no lograban emplearse y personas con bajos niveles académicos, que no aplican a muchas de las vacantes laborales del departamento, paulatinamente el moto-taxismo se fue extendiendo a 26 de los 32 departamentos del país (Osorio, 2010).

Yanez y Acevedo (2014) afirman que "en Colombia, el transporte de pasajeros en motocicletas se originó alrededor de los años ochenta en el corregimiento de Cotorra (Córdoba) en respuesta a la necesidad de transporte de la población de este corregimiento hasta la carretera que conducía a Lorica o Montería” (p. 37), por esto, el moto-taxismo se convirtió lentamente en una alternativa de ingresos económicos generalmente para muchas familias de escasos recursos económicos, a consecuencia de la inexistencia de fuentes de empleo formales, que brindaran una estabilidad económica. A pesar de ser una alternativa económica para muchas familias, este tipo de transporte informal se ha convertido en un serio problema para las autoridades nacionales, regionales y locales debido a la espera de pasajeros, ocupando espacio vial y peatonal, alto grado de accidentalidad y seguridad, pues en algunos casos propicia la delincuencia, sumado a la ilegalidad de tránsito vehicular por no contar con los documentos legales obligatorios de la motocicleta.

El periódico el Colombiano citado por Vargas, Tróchez y Tequin (2018) relata que "el Ministerio de Transporte, como ente rector de la política pública de transporte, no incluyó dentro del Plan Nacional de Desarrollo (PND), la habilitación del bicitaxismo y el mototaxismo como medios de transporte público en el país" (p. 208). De igual manera el periódico El Universal citado por Vargas et al. (2018) afirma que "el mototaxismo es un servicio de transporte ilegal en ciudades con más de 350.000 habitantes, sin embargo, hay 10 capitales de departamento en Colombia que tienen más de 4.000 mototaxis rodando en sus calles” (p. 208).

Esta actividad generalmente es realizada en ciudades pequeñas, sin embargo, en ciudades grandes como capitales de departamentos, esta actividad también se ejerce. Dentro de las normas legislativas el moto-taxismo es considerado un transporte informal pues no cuenta con los permisos necesarios para la utilización de las motocicletas como transporte público, tal como lo afirma Cogollo, Palomo y Guardela (2015): "El término Transporte Informal hace referencia a cualquier tipo de vehículo que se dedique a la prestación del servicio sin ningún tipo de seguimiento a las normas que regulan la actividad" (p. 17).

A pesar de los beneficios que representa para diversas personas el mototaxismo, en este momento es considerada una actividad ilegal, pues en el artículo 6 del decreto 170 
de 2001, se estipula que el Servicio Público de Transporte Terrestre Automotor Colectivo de Pasajeros es aquel que se presta bajo la responsabilidad de una empresa de transporte legalmente constituida y debidamente habilitada en esta modalidad, a través de un contrato celebrado entre la empresa y cada una de las personas que han de utilizar el vehículo de servicio público a esta vinculado, para recorrer total o parcialmente una o más rutas legalmente autorizadas, y en el artículo 12 del decreto 170 de 2001, establece habilitación a las empresas legalmente constituidas, interesadas en prestar el Servicio Público de Transporte Terrestre Colectivo de Pasajeros en el radio de acción Metropolitano, Distrital y Municipal deberán solicitar y obtener habilitación para operar.

Según la Alcaldía de Ocaña (2016), en el municipio circulan cerca de 3.500 a 5.000 motocicletas, las cuales son utilizadas para ejercer la labor del moto-taxismo, gran parte de estas cifras de motocicletas, pertenecen a personas que provienen de municipios aledaños y de otras regiones del país, sin tener en cuenta la alta cantidad de migrantes venezolanos que en los últimos años vienen ejerciendo esta labor.

Ocaña, no ha sido ajena a toda esta actividad de carácter ilegal, viendo como a través de los años las personas que se dedican a esta labor han aumentado considerablemente, creando problemáticas como las generadas en otras ciudades del país como ocupación del espacio público, accidentalidad, inseguridad, economías ilegales informales. Además, ha generado una competencia inequitativa con las empresas de transporte público legales que existen en la ciudad llevándolas al borde de la quiebra (La Opinión, 2017).

Ocaña siendo una ciudad de la región del Catatumbo, ha estado inmersa históricamente en el conflicto armado. Por tal razón, su economía ha estado ligada a mercados ilegales que han generado flujos de dinero los cuales dependen del cultivo de la coca, su cercanía con Venezuela generó que el comercio sea casi es su totalidad de productos contrabandeados, lo cual es una causal de desaparición de negocios previamente establecidos, así como disminución de actividades económicas formales y legales de la ciudad.

El panorama anterior convierte al moto-taxismo en una opción para muchos habitantes de la ciudad frente a las pocas oportunidades que se pueden encontrar en la ciudad en lo que se refiere a actividades formales y legales, sumado a esto no se observan iniciativas claras por parte de las autoridades locales en los últimos periodos de gobierno para dar una solución a esta problemática o tratar de minimizar el impacto en la ciudad. Amor (2017) sustenta:

Colombia no fue producto de un proyecto moderno de nación sino el resultado de una conquista colonial y una modernidad hecha a nuestro estilo. Por eso es que muchos procesos de modernidad se encuentran a medio camino o tomaron caminos impensados. El caso del mototaxismo, por ejemplo, se sitúa en el panorama de una Colombia que busca el lucro económico por encima de todo valor, no resuelve los problemas de movilidad, tampoco asume lo público como lo de todos, pero aún más interesante es un campo donde se expresa culturalmente los juegos de poder, lucha de clases y modelo de modernidad practicado" (p. 78).

El moto-taxismo es una actividad bastante compleja pues para algunos de los que ejercen esta labor, la única alternativa de ingresos económicos y por lo tanto subsistencia de sus familias son los conseguidos a raíz de esta actividad; convirtiéndose en un sector que genera dinámicas económicas muy importantes en la ciudad de Ocaña; sin embargo, la connotación de ilegalidad de esta actividad lleva a que sea clandestina y perseguida por 
las autoridades. El dilema es taxativo en la medida que es una fuente de empleo para los estratos menos favorecidos, pero es una actividad ilegal; por consiguiente, este trabajo de investigación presenta unos resultados con los que se espera se considere como pertinente dentro de los factores de desarrollo de instituciones de naturaleza social, y sean incluidas en las políticas gubernamentales que aborden el problema desde su complejidad socioeconómica y no simplemente desde una actividad legal o ilegal.

\section{Metodología}

Para la investigación se utilizó una metodología cuantitativa no experimental de tipo descriptivo, que permite el análisis de datos, dando así bases para sustentar la teoría planteada a través de técnicas estadísticas. Todo esto con el fin de especificar las propiedades, las características y los perfiles del grupo de mototaxistas que se sometieron al análisis, ya que para el caso de la investigación, la investigación pretende a través de la cuantificación entender la realidad del mototaxismo en la ciudad de Ocaña, la cual según Abalde y Muñoz-Cantero (1992) aseguran:

La metodología cuantitativa concibe el objeto de estudio como externo en un intento de lograr la máxima objetividad. Su concepción de la realidad coincide con la perspectiva positivista. Es una investigación normativa, cuyo objetivo está en conseguir leyes generales relativas al grupo (p. 94).

Las técnicas de recolección de datos aplicada fue la observación directa a través de los investigadores. Se realizó un conteo de los moto-taxistas; se tomaron los días donde más afluencia de tráfico había que fueron los días lunes y viernes y las horas pico que iban desde las 5 y 45 am a las 7 y 45 am, desde las 11 y 15 am a las 2 y 15 pm, y de las 4 y 45 pm a las 6 y 45 pm respectivamente. Explicar en detalle y rigurosidad científica la sistematización procedimental, ¿cuáles son las técnicas de recolección y procesamiento de la información? ¿Cuáles son los instrumentos que se utilizaron para recoger la información? ¿Cuál es la población? ¿Cuál es la muestra? ¿Qué criterios utilizan para incluir los sujetos en la muestra?

La metodología utilizada para el conteo de moto-taxistas fue por medio de la identificación de características o acciones particulares realizadas por ellos, algunas de las características principales es su vestuario o indumentaria (ropa que cubre casi la totalidad de su cuerpo), para evitar daños en la piel por las largas horas expuestos al sol, de igual manera se identificó indumentaria y accesorios como guantes, gafas de sol y un casco adicional, el cual entregan a su pasajero a la hora de montarse en la motocicleta. También se observan características particulares como la forma de ofrecer su servicio de transporte, haciendo alucón a su medio de transporte, "moto, moto" una frase peculiar, permite identificar al moto-taxista de cualquier otra persona que se transporte de igual manera en motocicleta.

\section{RESUlTAdos Y DISCUSióN}

Se tomaron 7 puntos de observación, los cuales se promediaron con los conteos realizados en cada punto, se aplicó la forma probabilística para encuestas con un margen de error para 7 y un nivel de confianza del 95\%, para un total de 187 encuestas. 
TABLA 1.

Resultado de los conteos

\begin{tabular}{|c|c|c|c|c|c|c|c|c|c|}
\hline \multirow[t]{5}{*}{ PUNTO } & \multicolumn{6}{|c|}{ HORARIOS } & \multirow{5}{*}{$\begin{array}{l}\text { Promedio } \\
\text { por punto }\end{array}$} & \multirow{5}{*}{$\begin{array}{l}\text { Porcentaje } \\
\text { según } \\
\text { población }\end{array}$} & \multirow{4}{*}{$\begin{array}{c}\text { Numero } \\
\text { de } \\
\text { encuestas } \\
\text { por }\end{array}$} \\
\hline & \multicolumn{3}{|c|}{ LUNES } & \multicolumn{3}{|c|}{ VIERNES } & & & \\
\hline & $5: 45$ & $11: 15$ & $4: 45$ & $5: 45-$ & $11: 15$ & $4: 45 p$ & & & \\
\hline & a & a & $\mathrm{p}$ & $7: 45$ & a & 6:45 pm & & & \\
\hline & $\begin{array}{c}7: 45 \\
\text { am }\end{array}$ & $\begin{array}{c}2: 15 \\
\mathrm{pm}\end{array}$ & $\begin{array}{l}6: 45 \\
\text { pm }\end{array}$ & $\mathrm{am}$ & $\begin{array}{c}2: 15 \\
\text { pm }\end{array}$ & & & & punto \\
\hline 1 & 601 & 664 & 503 & 622 & 590 & 702 & 613,6666667 & 15,76266107 & 29 \\
\hline 2 & 630 & 1304 & 1115 & 737 & 1321 & 1220 & 1054,5 & 27,08591977 & 52 \\
\hline 3 & 445 & 590 & 630 & 410 & 608 & 636 & 553,1666667 & 14,20865619 & 27 \\
\hline 4 & 452 & 490 & 271 & 327 & 530 & 458 & 421,3333333 & 10,8223811 & 20 \\
\hline 5 & 365 & 340 & 435 & 290 & 365 & 505 & 383,3333333 & 9,846311914 & 18 \\
\hline 6 & 382 & 530 & 354 & 375 & 755 & 412 & 468 & 12,02106255 & 23 \\
\hline \multirow[t]{2}{*}{7} & 278 & 610 & 297 & 292 & 632 & 286 & 399,1666667 & 10,25300741 & 18 \\
\hline & & & & & & $\begin{array}{c}\text { Total } \\
\text { mototaxistas }\end{array}$ & 3893,166667 & & \\
\hline
\end{tabular}

Fuente: Elaboración propia.

TABLA 2.

Edad/años

\begin{tabular}{|c|c|c|}
\hline Edad/años & Personas encuestadas & Porcentaje (\%) \\
\hline $19-23$ & 39 & 20.8 \\
\hline $24-28$ & 37 & 19.7 \\
\hline $29-33$ & 39 & 20.8 \\
\hline $34-38$ & 30 & 16.4 \\
\hline $39-43$ & 17 & 9 \\
\hline Mayor de 43 & 25 & 13.3 \\
\hline Total & 187 & 100 \\
\hline
\end{tabular}

Fuente: Elaboración propia.

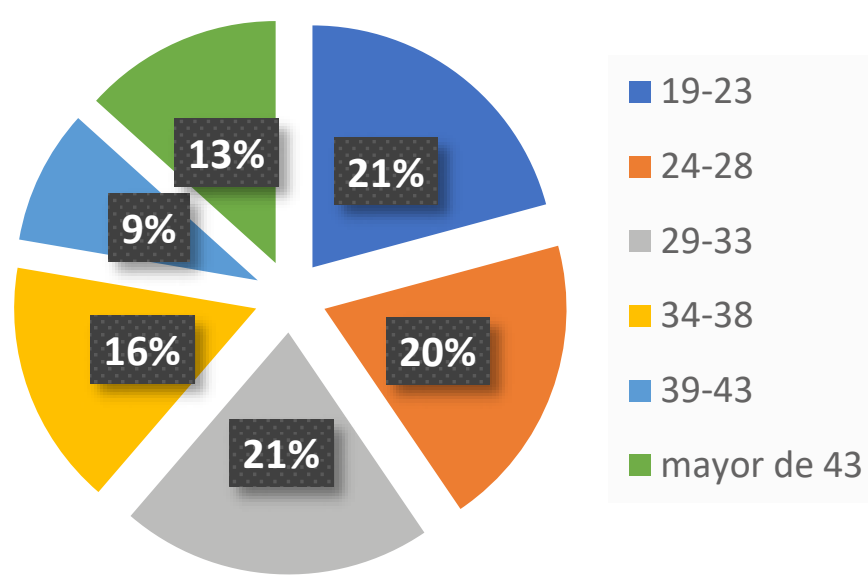

Figura 1. Edad/años

Fuente: Elaboración propia. 
El promedio de edades de personas dedicadas al moto-taxismo de 187 encuestados, comienzan a partir de los 19 años, encontrando diferentes edades, incluyendo mayores de 43 años. Se encuentra que el 13\% de personas dedicadas al moto-taxismo son personas mayores de 43 años, los intervalos de edades que representan un valor significativo, son los que tienen entre 19-23 y 29-33 años, correspondiendo así a un 21\%, de igual manera, el intervalo de edades entre los 24-28, representa el 20\%. Las demás edades, 34-38 y 39-43, representan el 16\% y 9\%, respectivamente, encontrando así, que gran parte de la población joven de la región se dedica a esta labor.

TABLA 3.

Estrato social

\begin{tabular}{|c|c|c|}
\hline Estrato social & Personas encuestadas & Porcentaje (\%) \\
\hline 1 & 134 & 71.3 \\
\hline 2 & 41 & 21.9 \\
\hline 3 & 10 & 5.3 \\
\hline 4 & 2 & 1 \\
\hline Total & 187 & 100 \\
\hline
\end{tabular}

Fuente: Elaboración propia.

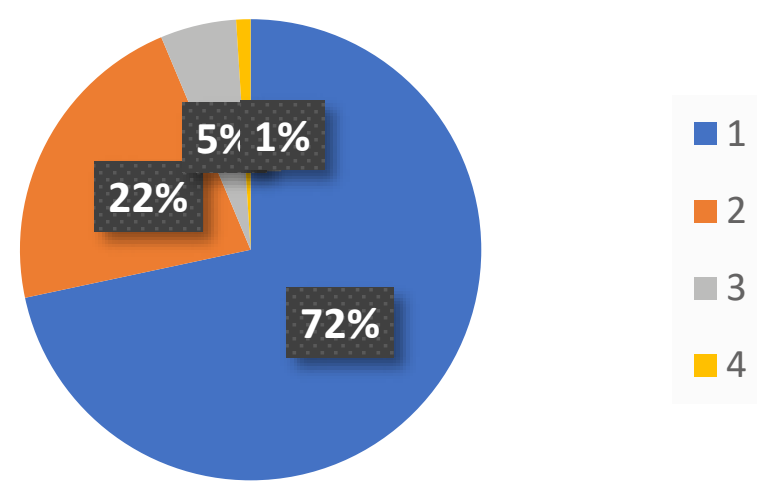

Figura 2. Estrato social

Fuente: Elaboración propia.

La gran mayoría de los encuestados se encuentran dentro de los estratos sociales 1 y 2 , son las personas de más escasos recursos económicos, los cuales encuentran en el mototaxismo una forma de generar ingresos y llevar el sustento diario a sus hogares.

TABla 4.

Estado civil

\begin{tabular}{llll}
\hline \multicolumn{1}{c}{ Estado civil } & & Personas encuestadas & Porcentaje (\%) \\
\hline Soltero & 81 & 43.3 \\
Casado & 96 & 51.4 \\
Divorciado & 10 & 5.3 \\
Total & 187 & 100 \\
\hline
\end{tabular}

Fuente: Elaboración propia. 


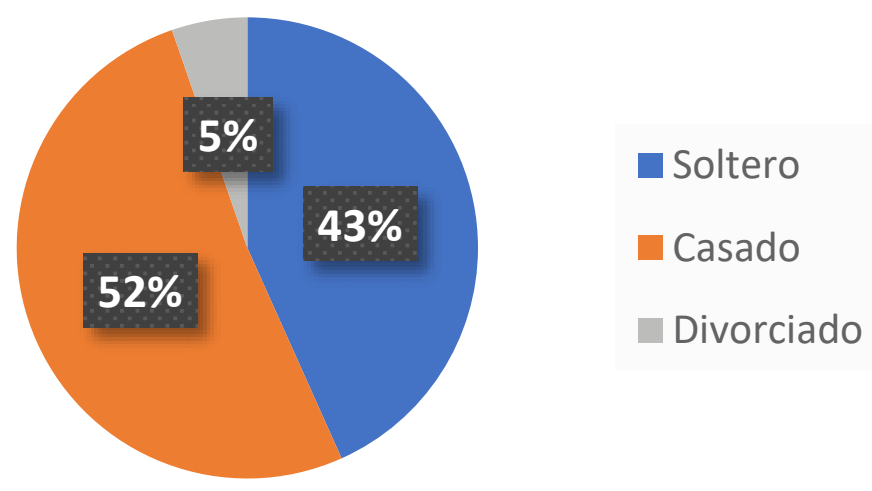

Figura 3. Estado civil

Fuente: Elaboración propia.

El estado civil de los moto-taxistas es directamente proporcional con la edad de los mismos ya que un $43 \%$ son solteros y su edad oscila entre los 19 a los 23 años y un 51\% son casados o viven en unión libre, y su edad va desde los 24 a los 33 años aproximadamente.

TABLA 5.

Personas que dependen de su labor

\begin{tabular}{lll}
\hline \multicolumn{1}{c}{ Personas que dependen su labor como moto-taxista } & Personas encuestadas & Porcentaje (\%) \\
\hline Ninguna & 15 & 8.1 \\
Entre 1 y 5 personas & 170 & 90.9 \\
Más de 5 personas & 2 & 1 \\
Total & 187 & 100 \\
\hline
\end{tabular}

Fuente: Elaboración propia.

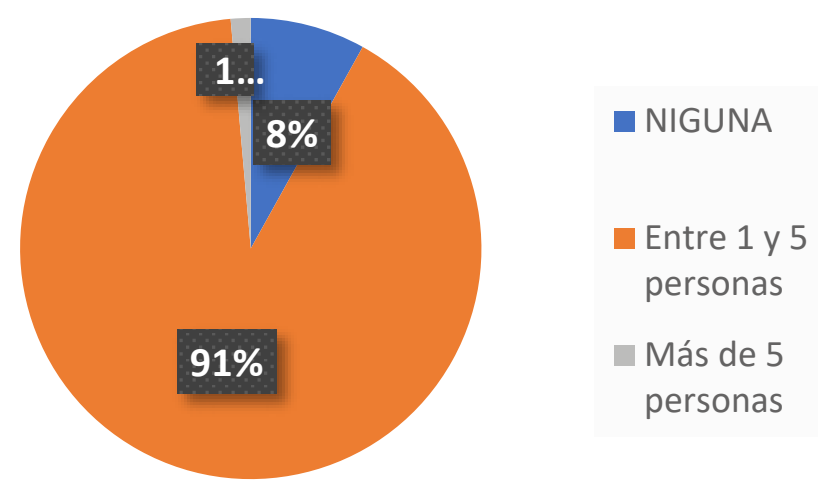

Figura 4. Personas que dependen de su labor Fuente: Elaboración propia.

Ocaña es una población que cuenta con 128.742 habitantes; según las cifras obtenidas en las encuestas, 170 moto-taxistas cuentan con familias conformadas entre 1 y 5 personas, las cuales dependen de su labor, esto representa el 90\%, 2 de ellos tienen familias con más de 5 integrantes, los cuales también dependen de su labor, estos valores, son significativamente altos para depender solo de esta labor. Solo el 8\% de la población encuestada, no tienen familia de la que dependa su labor. 
TABLA 6.

Nivel de escolaridad

\begin{tabular}{llll}
\hline \multicolumn{1}{c}{ Nivel de escolaridad } & \multicolumn{2}{c}{ Personas encuestadas } & Porcentaje (\%) \\
\hline Primaria no terminada & 24 & 12.8 & 17.1 \\
Primaria terminada & 32 & 29.4 \\
Secundaria no terminada & 55 & 34.7 \\
Secundaria terminada & 65 & 5.8 \\
Universidad no terminada & 11 & 0 \\
Universidad terminada & 0 & 100 \\
Total & 187 &
\end{tabular}

Fuente: Elaboración propia.

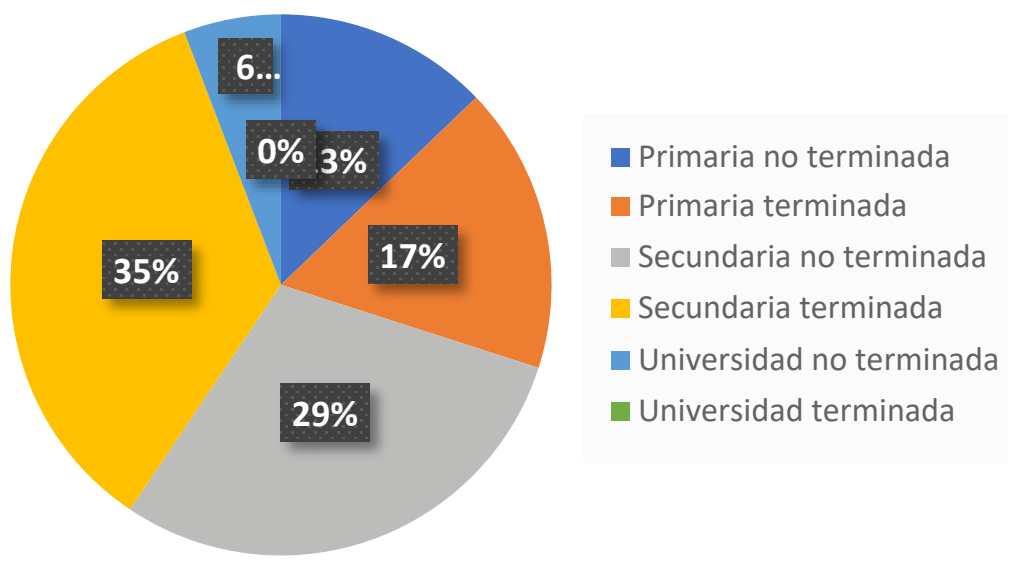

Figura 5. Nivel de escolaridad Fuente: Elaboración propia.

En este punto se puede evidenciar un alto índice de deserción estudiantil ya que el 29\% no terminó sus estudios de secundaria. El 35\% de los encuestados terminaron sus estudios de secundaria, pero la falta de oportunidades laborales, el limitado acceso a la educación superior lleva a estos jóvenes a ejercer la labor del moto-taxismo. El porcentaje más alto son las personas de menor edad dentro de los encuestados. Según el plan de desarrollo de Ocaña de los 29.576 estudiantes en edad escolar solo se encuentran matriculados 19.152 estudiantes y que los jóvenes no matriculados son: el $23 \%$ no cursan preescolar, el $21 \%$ no cursan básica primaria, el 33\% no cursan básica secundaria y el 65\% no cursan educación media, porcentajes altos que se relacionan en la tabla anterior.

TABLA 7.

Hace cuantos años es moto-taxista

\begin{tabular}{lll}
\hline Hace cuantos años es moto-taxista & \multicolumn{1}{c}{ Personas encuestadas } & Porcentaje (\%) \\
\hline Entre 1 y 3 & 100 & 53.4 \\
Entre 4 y 6 & 37 & 19.7 \\
Más de 6 & 50 & 27.7 \\
Total & 187 & 100 \\
\hline
\end{tabular}

Fuente: Autoría propia 


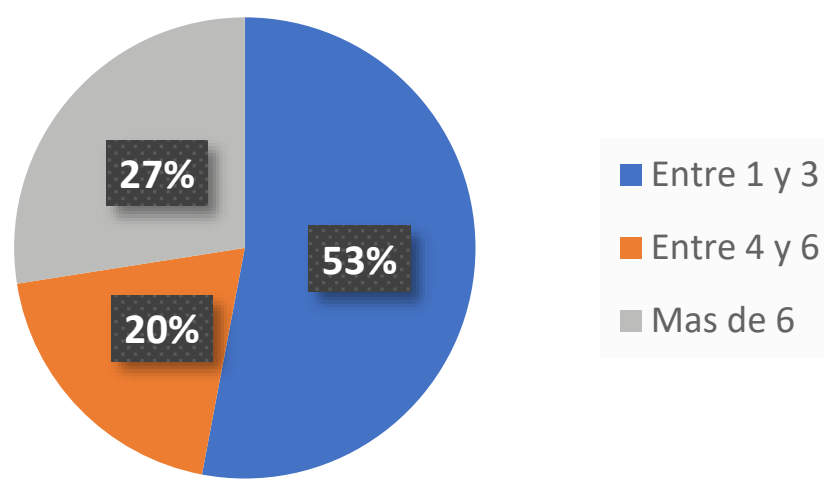

Figura 6. hace cuantos años es moto-taxista Fuente: Elaboración propia.

Como se puede observar en la figura 6. los últimos tres años se viene incrementando este fenómeno del moto-taxismo ya que el $53 \%$ de encuestados está ejerciendo esta actividad entre uno y tres años de antigüedad, algunas posibles causas pueden ser las bajas fuentes de empleo, el acceso a una educación superior, la situación que se presenta con Venezuela repercute en esta variable ya que muchas personas, realizaban sus labores con productos de origen venezolano pero debido a la crisis económica y social que el país vecino está viviendo tuvieron que dejar de ejercer sus labores y dedicarse al moto-taxismo.

TABLA 8.

Razones para incorporarse a la actividad

\begin{tabular}{lll}
\hline \multicolumn{1}{c}{ Razones para incorporarse a la actividad } & Personas encuestadas & Porcentaje (\%) \\
\hline Falta de oportunidad laboral & 27 & 14.4 \\
Necesidad & 41 & 21.9 \\
Desempleo & 106 & 56.6 \\
Desplazamiento o inmigración & 4 & 2.1 \\
Trabajo fácil & 9 & 4.8 \\
Total & 187 & 100 \\
\hline
\end{tabular}

Fuente: Elaboración propia.

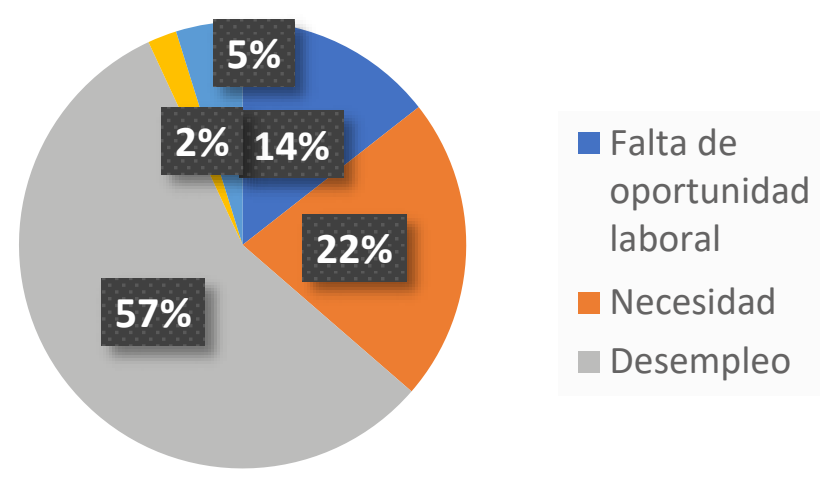

Figura 7. Razones para incorporarse a la actividad Fuente: Elaboración propia. 
El desempleo la mayor razón para iniciar la actividad del moto-taxismo, la falta oportunidades laborales obliga a estas personas a ejercer esta actividad dentro de los 3893 moto-taxistas el $57 \%$ se encuentra sin un trabajo formal lo que equivale a 2219 personas obligadas a realizar esta labor.

TABLA 9.

Cilindraje de la motocicleta

\begin{tabular}{lll}
\hline \multicolumn{1}{c}{ Cilindraje de la motocicleta } & \multicolumn{1}{c}{ Personas encuestadas } & Porcentaje (\%) \\
\hline 100 & 39 & 20.8 \\
110 & 44 & 23.5 \\
115 & 11 & 5.8 \\
125 & 67 & 35.8 \\
135 & 6 & 3.2 \\
150 & 20 & 10.7 \\
Total & 187 & 100 \\
\hline
\end{tabular}

Fuente: Elaboración propia.

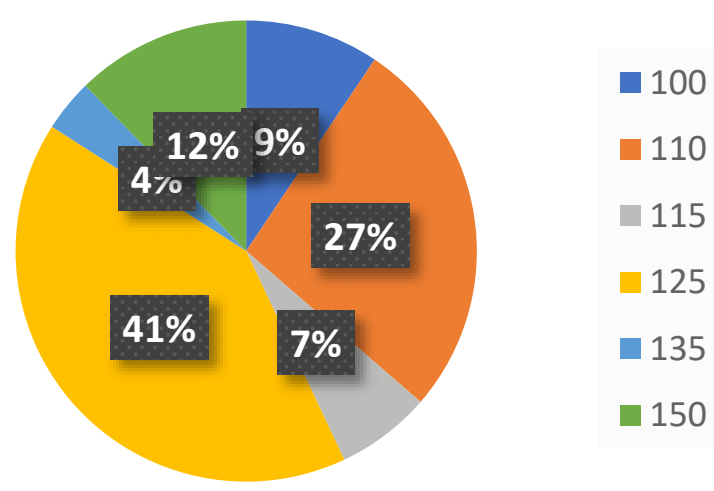

Figura 8. Cilindraje de la motocicleta Fuente: Elaboración propia.

El cilindraje de la motocicleta que predomino fue el de $125 \mathrm{cc}$, ya que son las motocicletas de mayor comercialización y se adaptan mejor al trabajo del moto-taxismo, ya sea por su valor monetario, por su bajo consumo de combustible o por la economía en sus refacciones. Estas características de las motocicletas llevan a que los ingresos de los mototaxistas puedan ser mayores

TABla 10.

Cuantos tiempos es la motocicleta

\begin{tabular}{|c|c|c|}
\hline Cuantos tiempos es la motocicleta & Personas encuestadas & Porcentaje (\%) \\
\hline $2 \mathrm{t}$ & 46 & 24.6 \\
\hline $4 \mathrm{t}$ & 141 & 75.4 \\
\hline Total & 187 & 100 \\
\hline
\end{tabular}

Fuente: Elaboración propia. 


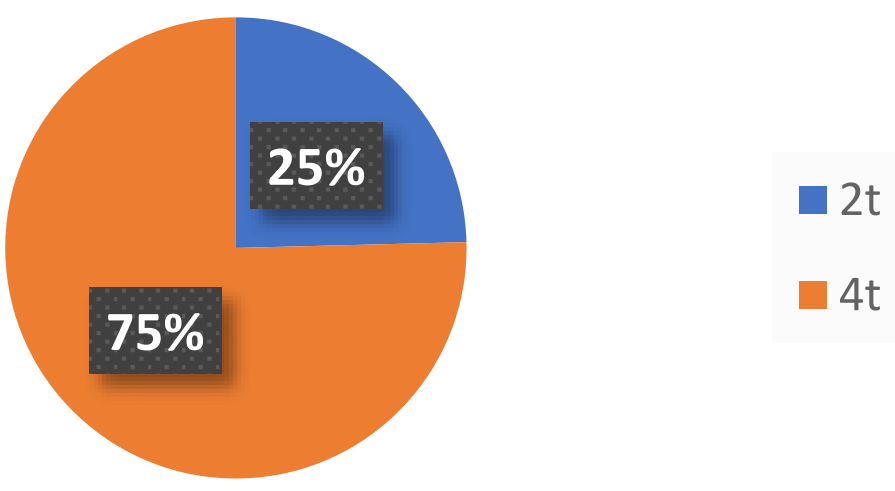

Figura 9. Cuantos tiempos es la motocicleta Fuente: Elaboración propia.

Las motocicletas de cuatro tiempos son las más adecuadas para ejercer este trabajo debido a una característica primordial que es su bajo consumo de gasolina respecto a las motocicletas de dos tiempos y las motocicletas de dos tiempos deben utilizar dos tipos de aceite para su correcto funcionamiento; mientras que las motocicletas de cuatro tiempos solo utilizan un tipo de aceite el cual es un ahorro de dinero para el moto-taxista.

TABLA 11.

Nacionalidad de la motocicleta

\begin{tabular}{lll}
\hline \multicolumn{1}{c}{ Nacionalidad de la motocicleta } & Personas encuestadas & Porcentaje (\%) \\
\hline Colombiana & 144 & 77.1 \\
venezolana & 43 & 22.9 \\
Total & 187 & 100 \\
\hline
\end{tabular}

Fuente: Elaboración propia.

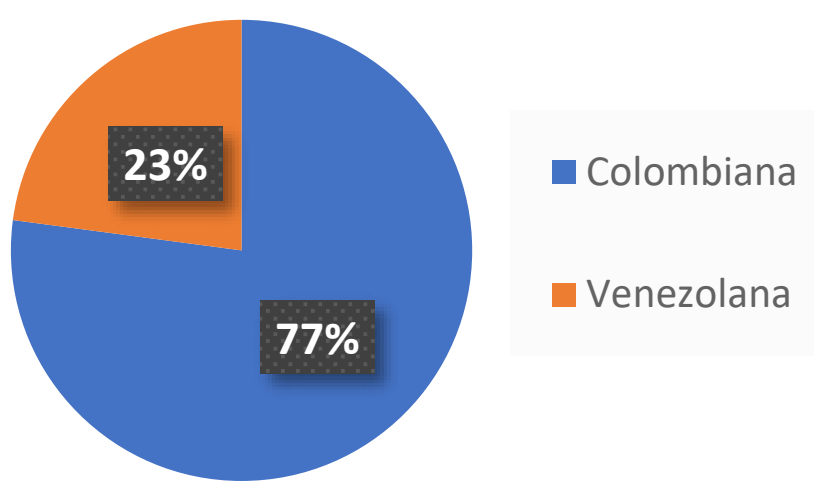

Figura 10. nacionalidad de la motocicleta Fuente: Elaboración propia.

Norte de Santander por ser un departamento fronterizo, permite la movilización de vehículos de origen venezolano de las 3893 motocicletas, 895 son venezolanas lo que equivalen al $23 \%$ de la totalidad de encuestados.

TABla 12. 


\section{Propiedad de la motocicleta}

\begin{tabular}{lll}
\hline Propiedad de la motocicleta & \multicolumn{1}{c}{ Personas encuestadas } & Porcentaje (\%) \\
\hline Propia & 148 & 79.1 \\
Familiar & 23 & 12.3 \\
Alquiler & 13 & 6.9 \\
Otro & 3 & 1.6 \\
Total & 187 & 100 \\
\hline
\end{tabular}

Fuente: Elaboración propia.

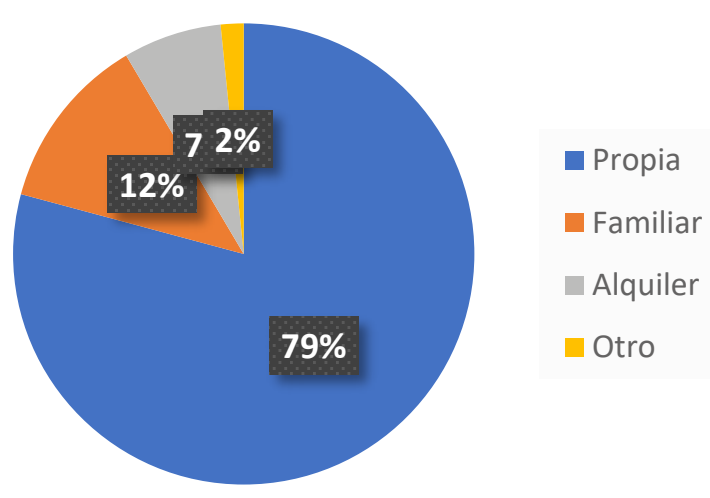

Figura 11. Propiedad de la motocicleta Fuente: Elaboración propia.

Las motocicletas utilizadas para realizar esta labor, el 79\% son de propiedad del mototaxista ya que siendo propias van a generar mayores ingresos, el $12 \%$ son de propiedad familiar, lo que permite de igual manera, que los ingresos sean totales, el 8.5\% corresponde a motocicletas alquiladas o de otro tipo, lo que disminuye los ingresos, pues gran parte de estos se van al pago del alquiler.

Otra variable estudiada fue el ingreso que obtenían en promedio, el cual fue de $\$ 35.000$ COP diarios, si se multiplica por 3893 moto-taxistas contados; el ingreso total diario de estos sería de \$136.255.000 COP. Mensualmente los moto-taxistas estarían generando $\$ 4.087 .650 .000 \mathrm{COP}$ un monto bastante significativo

En lo que concierne al consumo de combustible por parte de las motocicletas, estas consumen un galón de gasolina diario, el cual tiene un costo de $\$ 7800 \mathrm{COP}$, si se multiplican por el número de motocicletas, serian 3893 galones de gasolina diarios, el cual tienen un costo de $\$ 30.365 .400$ COP. Mensualmente los moto-taxistas le están generando al municipio $\$ 910.962 .000 \mathrm{COP}$.

En los últimos años en el Municipio de Ocaña el fenómeno del moto-taxismo ha venido creciendo desproporcionadamente, considerado este trabajo informal e ilegal, debido a que los vehículos no son los adecuados para ejercer la labor de un transporte legal y debidamente constituido. Cabe destacar que, en Ocaña, es un municipio donde las fuentes de ingreso y las oportunidades laborales son muy bajas, pues no se cuentan con empresas o entidades que generen un gran número de personas para emplearlas, de igual manera la economía informal que se vive en Ocaña, permite la subsistencia de manera ilegal. 
Otro punto de gran importancia en esta investigación es el mal estado y la ausencia de vehículos de empresas prestadoras de servicios públicos que lleguen a todos los barrios de la ciudad, la mayoría de las rutas que cubre el transporte público no llega o pasa por los barrios periféricos de la región, lo que obliga a los habitantes de la ciudad a tomar el moto-laxismo como su medio de transporte.

El moto-taxismo genera altos índices de personas que se dedican a este al trabajo, el cual se ha convertido en una problemática social y que el gobierno local, departamental y nacional poco han hecho para frenar esta fenómeno, una de las variables que se pudo analizar en esta investigación fue el flujo de dinero que esta actividad genera y el aporte positivo a la economía del municipio, la encuesta realizada arrojo que diariamente esta actividad genera $\$ 136.225 .000 \mathrm{COP}$ aproximadamente y mensualmente $\$ 4.087 .650 .000$ COP, flujos de dinero sumamente altos, un consumo de gasolina de 3893 galones diarios lo que equivalen a $\$ 30.365 .400 \mathrm{COP}$, aproximadamente y mensualmente $\$ 910.962 .000 \mathrm{COP}$ a pesar de ser un trabajo ilegal, indirectamente genera ganancias al municipio y le aporta positivamente a la economía.

En la investigación se pudo evidenciar que de esta actividad dependen muchas familias ya que un $51 \%$ de los moto-taxistas son casados y que en un $90 \%, 3$ personas en promedio dependen económicamente del moto-taxista. El total de personas que dependen de esta labor equivalen al 9\% de la población total del municipio lo cual es un valor significativo, se debe tener en cuenta que la población en su mayoría son de estratos sociales 1 y 2 , los jóvenes que no terminaron y los que terminaron su educación secundaria son los que en su mayoría están ejerciendo esta labor, una cifra bastante amplia lo que es un panorama un poco turbio para los jóvenes de la ciudad que no encuentran otra fuente de trabajo y de generar ingresos más que con esta labor.

Esta actividad considerada una problemática tiene un trasfondo social y económico bastante complejo y requiere un acompañamiento más eficaz por parte del estado. El mototaxismo es una alternativa de subsistencia para las personas de estratos sociales menos favorecidos y genera una dinámica económica muy importante en la ciudad, es un problema debido a su causal de informalidad, pero a su vez es una fuente de empleo para las personas de más escasos recursos económicos y la mayoría de desempleados que encuentran en el moto-taxismo una manera de subsistir.

\section{Conclusiones}

El objetivo del artículo se centró en analizar las causas y consecuencias que llevan a gran cantidad de habitantes de Ocaña a ejercer esta actividad; partiendo de un censo poblacional de las personas que la ejercen, promedio de las ganancias diarias, edades, estrato social, nivel educativo, núcleo familiar, entre otros, y analizando la relevancia que tiene el mototaxismo en la economía de la ciudad.

A pesar de que Ocaña es un municipio pequeño, que cuenta con 128.742 habitantes, es un municipio donde el transporte ilegal moto-taxismo representa en gran medida, un porcentaje significativo de los ingresos económicos de los habitantes. Muchas de las personas que se dedican a esta labor, lo hacen por la falta de oportunidades laborales en la región, y encuentran en este oficio, el sustento económico para su familia. En el estudio realizado, 
se evidenció que gran parte de la población Ocañera dedicada al moto-taxismo, conforman familias numerosas, siendo estas la mayoría entre 1 y 5 miembros, los cuales dependen en su mayoría de esta labor. El moto-taxismo se convirtió en la oportunidad laboral para muchas personas que no cuentan con la preparación necesaria para postularse a un trabajo que le brinde las condiciones económicas necesarias para abastecer su familia, también es la salida laboral para muchos profesionales que no encuentran un trabajo fijo, y para las personas que por su edad ya no aplican a un empleo formal.

Las ganancias diarias obtenidas gracias al moto-taxismo, muchas veces superan el valor de un trabajo diario remunerado, esta es otra razón por la que tantas personas se dedican a esta labor, tiendo en cuenta, que no manejan horarios y su tiempo disponible es de acuerdo a sus ganancias.

A pesar de que el moto-taxismo es un transporte ilegal, el impacto socioeconómico que esta labor causa en Ocaña es grande, es el sustento económico de muchas familias, pero a raíz de este, el consumo de gasolina deja grandes ganancias, pues como se dijo anteriormente, mensualmente aporta $\$ 910.962 .000 \mathrm{COP}$ en consumo de gasolina, en un total de 3893 moto-taxistas contados.

\section{REFERENCIAS}

Abalde, E. y Muñoz-Cantero, J. M. (1992). Metodología cuantitativa vs cualitativa. En, J. Muñoz y E. Abalde (Coord.), Metodología educativa I. La Coruña: Universidade da Coruña.

Alcaldía de Ocaña. (2016). Plan de desarrollo es la hora de Ocaña 2016-2019. [Online]. Recuperado de https://ocananortedesantander.micolombiadigital.gov.co/sites/ocananortedesantander/content/files/000108/5376_plandedesarrolloeslahoradeocaa20162019.pdf

Amor, M. P. (2017). La historia triple del mototaxismo: ¿Plaga, contrapoder o negocio? [Trabajo grado]. Universidad Tecnológica Cartagena de Indias, Cartagena, Colombia.

Caro, L. P. y Cotinchara, L. J. (2018). Caracterización del Perfil Socioeconómico del Gremio de los Mototaxista del Municipio de San Martín de los Llanos. [Tesis grado]. Universidad de Los Llanos, Villavincencio, Colombia. Disponible en https://repositorio.unillanos.edu.co/bitstream/001/1109/1/RUNILLANOS\%20ECO\%200420\%20CARACTERIZACION\%20DEL\%20PERFIL\%20SOCIECONOMICO\%20DEL\%20GREMIO\%20 DE\%20LOS\%20MOTOTAXISTAS\%20DEL\%20MUNICIPIO\%20DE\%20SAN\%20MARTIN\%20DE\%20LOS\%20LLANOS.pdf

Cogollo, J. D. J., Palomo, Y. E. y Guardela, P. A. (2015). Efectos del mototaxismo en el transporte público colectivo y la movilidad urbana en las rutas: 2, 3A, 4, 6, 7, 31, 35, Bayunca. [Tesis grado]. Universidad de Cartagena, Cartagena, Colombia. Disponible en http://hdl.handle.net/11227/2232

La Opinión. (octubre 20, 2017). Transporte legal en Ocaña protesta por competencia pirata. La Opinión. Disponible en https://www.laopinion.com.co/ocana

Osorio, B. C. (2010). El mototaxismo ¿Problema informal o un medio de generación de ingresos para resolver en parte el problema del desempleo en montería? Económicas CUC, 31(1), 89-104. Disponible en https://revistascientificas.cuc.edu.co/economicascuc/ article/view/1185 
Rodríguez, J. P. y González, Y. M. (2017). La metáfora como mecanismo de variación léxico-semántica en el habla de los jóvenes mototaxistas del Carmen de Bolívar. [Trabajo grado]. Universidad de Cartagena, Cartagena, Colombia. Disponible en http://hdl. handle.net/11227/5580

Vargas, L. F., Tróchez, K. J. y Tequin, D. Y. (2018). Impacto económico, social y financiero que tiene el mototaxismo en la ciudad de Florencia Caquetá. FACE: Revista de la Facultad de Ciencias Económicas y Empresariales, 17(2), 206-216. Disponible en http:// revistas.unipamplona.edu.co/ojs_viceinves/index.php/FACE/article/view/2955

Yánez, M. y Acevedo, K. (2014). Determinantes de la decisión de los mototaxistas en cartagena (colombia) de infringir la medida de pico y placa. Revista Facultad de Ciencias Económicas, 22(1), 35-47. https://doi.org/10.18359/rfce.638

Fernando Barragan Sánchez es Zootecnista de la Universidad Francisco de Paula Santander (Colombia), enfocado hacia las produccion pecuaria. Profesional de apoyo del consultorio Sociambiental y agropecuario del Nororiente colombiano, brindando apoyo desde los procesos académicos a partir del reconocimiento y acompañamiento de las comunidades y sectores productivos, a través de la figura de consultoría, promoviendo la articulación institucional, el mejoramiento en los procesos técnico-productivos, impulsando el desarrollo sustentable y realizando proyección a la comunidad. https://orcid.org/0000-0002-7807-5411

Jose Manuel Alba Maldonado es Candidato a Doctor en Historia Social y Política, con formación de investigación con vision académica. Licenciado en Filosofía competente en docencia e investigación en los campos de la ética, la política y humanidades. Magister en Desarrollo Rural, con capacidad reflexiva de los modelos de desarrollo del país, desde la política y la economía. Ingeniería agrónomo, con profundización en medio ambiente, educación y gestión ambiental. https://orcid.org/0000-0002-5384-7606

Merely Zoraida Gelvez Santiago es Ingeniera Ambiental de la Universidad Francisco de Paula Santande (Colombia). Especialista en Gerencia Social de la Universidad Simón Bolívar (Colombia), enfocada hacia las zonas rurales de Colombia. Profesional de apoyo del consultorio Sociambiental y agropecuario del Nororiente Colombiano, brindando apoyo desde los procesos académicos a partir del reconocimiento y acompañamiento de las comunidades y sectores productivos. https://orcid.org/0000-0003-0450-4874 